DO-TH 00/02

hep-ph/0003149

\title{
Neutrino oscillation experiments and limits on lepton-number and lepton-flavor violating processes
}

\author{
Werner Rodejohann* \\ Lehrstuhl für Theoretische Physik III, \\ Universität Dortmund, Otto-Hahn Str. 4, \\ 44221 Dortmund, Germany
}

\begin{abstract}
Using a three neutrino framework we investigate bounds for the effective Majorana neutrino mass matrix. The mass measured in neutrinoless double beta decay is its (11) element. Lepton-number and -flavor violating processes sensitive to each element are considered and limits on branching ratios or cross sections are given. Those processes include $\mu^{-} e^{+}$conversion, $K^{+} \rightarrow \pi^{-} \mu^{+} \mu^{+}$or recently proposed highenergy scattering processes at HERA. Including all possible mass schemes, the three solar solutions and other allowed possibilities, there is a total of 80 mass matrices. The obtained indirect limits are up to 14 orders of magnitude more stringent than direct ones. It is investigated how neutrinoless double beta decay may judge between different mass and mixing schemes as well as solar solutions. Prospects of detecting processes depending on elements of the mass matrix are also discussed.
\end{abstract}

Keywords: Oscillation, Lepton number violation, Double beta decay

\footnotetext{
*Email address: rodejoha@dilbert.physik.uni-dortmund.de
} 


\section{Introduction}

In recent years overwhelming evidence for non-vanishing neutrino masses was collected in atmospheric [1, 2], solar [3] and accelerator [4] experiments, opening a window into a variety of new phenomena [5]. The results are interpreted in terms of neutrino oscillations governed by a mixing angle and a mass-squared difference. Analyzing the data of the respective experiments yields typically values of

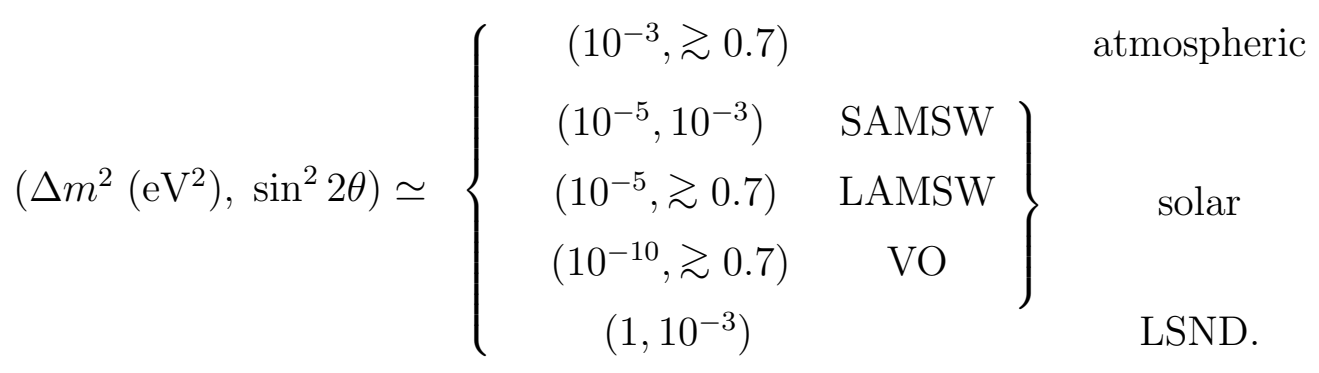

Here SAMSW (LAMSW) denotes the small (large) angle MSW [6] solution and VO the vacuum oscillation solution of the solar neutrino problem. One sees that the atmospheric and solar mass scale obey the following relation:

$$
\Delta m_{\odot}^{2} \ll \Delta m_{\mathrm{A}}^{2},
$$

regardless of the solar solution chosen. In order to avoid sterile neutrinos one usually leaves out the LSND [4 result (which also gives time to wait until the conflict with KARMEN [7] is resolved). We are thus working in a three neutrino framework, which allows a simple derivation of the leptonic Maki-Nakagawa-Sakata (MNS) matrix [8]. Only five mass schemes can accommodate the results in a three neutrino framework, three hierarchical and two degenerate schemes with the overall scale given by cosmological arguments to be of the order of a few eV. These mass schemes can be divided into two scenarios. In addition, we distinguish the cases in which one element of the mixing element is zero as indicated by $\mathrm{CHOOZ}$ data and the general case of all elements being non-zero. Ordering effects of some elements are also considered.

From the MNS matrix one can infer limits on the $3 \times 3$ matrix of effective Majorana neutrino masses defined as

$$
\begin{gathered}
\left\langle m_{\alpha \beta}\right\rangle=\left|\left(U \operatorname{diag}\left(m_{1}, m_{2}, m_{3}\right) U^{\mathrm{T}}\right)_{\alpha \beta}\right| \\
=\left|\sum m_{i} U_{\alpha i} U_{\beta i}\right| \leq \sum m_{i}\left|U_{\alpha i} U_{\beta i}\right| \text { with } \alpha, \beta=e, \mu, \tau,
\end{gathered}
$$

where $U$ is the mixing matrix and the $m_{i}$ are mass eigenvalues. With this approximation, $\left\langle m_{\alpha \beta}\right\rangle$ is symmetrical. The mass measured in neutrinoless double beta decay $(0 \nu \beta \beta)$ is the (ee) element of this matrix and has been considered by several authors [9, 10]. However, the complete matrix is rarely analyzed in terms of phenomenological consequences, except

for the elements $\left\langle m_{e \mu}\right\rangle$ and $\left\langle m_{\mu \mu}\right\rangle$ in Refs. [11], although without giving concrete numbers. We introduce processes dependent on every element of $\left\langle m_{\alpha \beta}\right\rangle$, like $\mu^{-} e^{+}$conversion, $K^{+} \rightarrow$ 
$\pi^{-} \mu^{+} \mu^{+}$or recently proposed high-energy scattering processes, finding rather discouraging results for branching ratios or life times. We compare our results (calling them indirect limits) with current experimental (direct) bounds and find that our limits are up to 14 orders of magnitude more stringent than current experimental data and therefore beyond experimental access in the near future. Special attention is paid to $0 \nu \beta \beta$ and its sensitivity on mass and mixing. Though it will be much easier to wait for further astrophysical data to distinguish between vacuum and MSW solutions for the solar neutrino problem, it would be a remarkable experiment to decide via terrestrial nuclear physics experiments if matter effects inside the Sun are of importance or not. Unfortunately, it turns out that the only scheme delivering values inside the range of next generation experiments is insensitive on the solar solutions.

The paper is organized as follows: In Section 2 we present the usual assumptions that lead to the derivation of the MNS matrix and $\left\langle m_{\alpha \beta}\right\rangle$. Section 3 gives the results and pays special attention to the $(e e)$ element of $\left\langle m_{\alpha \beta}\right\rangle$. In Section 4 processes are introduced which are sensitive on the respective elements of $\left\langle m_{\alpha \beta}\right\rangle$ just as $0 \nu \beta \beta$ is on $\left\langle m_{e e}\right\rangle$. Section 5 concludes the paper.

\section{Oscillation probabilities and mass schemes}

The phenomenology of neutrino oscillations is well reviewed in the literature, see e.g. [12]. Flavor eigenstates $\nu_{\alpha}(\alpha=e, \mu, \tau)$ are connected to mass eigenstates $\nu_{i}(i=1,2,3)$ via an unitary matrix, i.e. $\nu_{\alpha}=U_{\alpha i} \nu_{i}$. For Majorana neutrinos this matrix can be parametrized as [13]

$$
U_{\alpha i}=\left(\begin{array}{ccc}
c_{1} c_{3} & s_{1} c_{3} e^{i \lambda_{1}} & s_{3} e^{-i \delta} \\
\left(-s_{1} c_{2}-c_{1} s_{2} s_{3} e^{i \delta}\right) e^{-i \lambda_{1}} & c_{1} c_{2}-s_{1} s_{2} s_{3} e^{i \delta} & s_{2} c_{3} e^{i\left(\lambda_{2}-\lambda_{1}\right)} \\
\left(s_{1} s_{2}-c_{1} c_{2} s_{3} e^{i \delta}\right) e^{-i \lambda_{2}} & \left(-c_{1} s_{2}-s_{1} c_{2} s_{3} e^{i \delta}\right) e^{-i\left(\lambda_{2}-\lambda_{1}\right)} & c_{2} c_{3}
\end{array}\right)
$$

with $c_{i}=\cos \theta_{i}$ and $s_{i}=\sin \theta_{i}$. Three $\mathrm{CP}$-violating phases are present. For neutrino oscillations, only one phase $(\delta)$ contributes [14]. Effects of all phases are discussed e. g. in [13], for our estimations we shall skip them, since we are not interested in CP violation. In

addition, the probabilities we use depend only on the absolute values of the mixing matrix elements. The probability of a flavor state $\alpha$ to oscillate into a state $\beta$ is given by

$$
P_{\alpha \beta}=\delta_{\alpha \beta}-2 \operatorname{Re} \sum_{j>i} U_{\alpha i} U_{\alpha j}^{*} U_{\beta i}^{*} U_{\beta j}\left(1-\exp i \Delta_{j i}\right)
$$

Here

$$
\Delta_{j i}=\frac{L}{2 E} \Delta m_{j i}^{2}=2.54 \frac{L / \mathrm{km}}{E / \mathrm{GeV}} \Delta m_{j i}^{2} / \mathrm{eV}^{2} \text { with } \Delta m_{j i}^{2}=m_{j}^{2}-m_{i}^{2} .
$$

Without loss of generality we assume

$$
m_{3}>m_{2}>m_{1}>0 \text {. }
$$




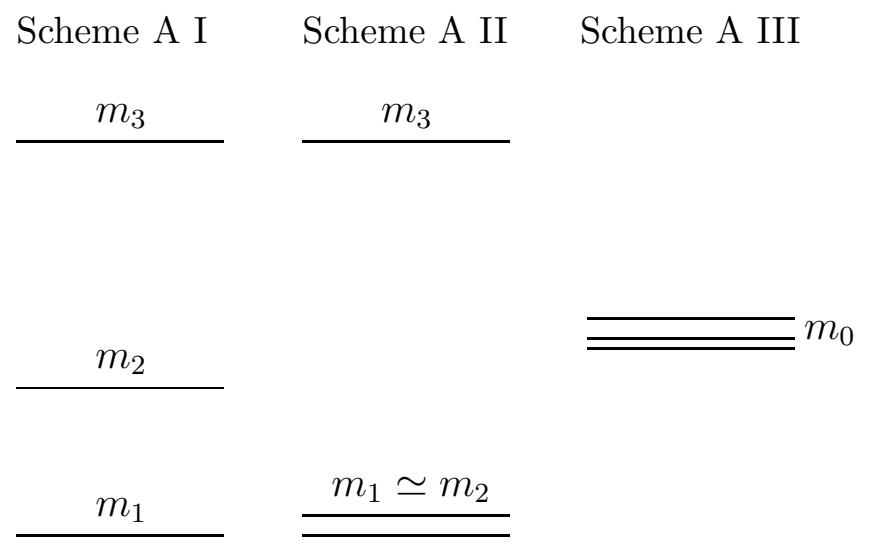

Figure 1: The three mass schemes that can accommodate the relation $\Delta m_{21}^{2} \ll \Delta m_{31}^{2} \simeq$ $\Delta m_{32}^{2}$.

With the above relation there are two possibilities to accommodate $\Delta m_{\odot}^{2} \ll \Delta m_{\mathrm{A}}^{2}$ :

$$
\begin{array}{ll}
\text { Scenario A : } & \Delta m_{21}^{2}=\Delta m_{\odot}^{2} \ll \Delta m_{\mathrm{A}}^{2}=\Delta m_{31}^{2} \simeq \Delta m_{32}^{2} \\
\text { Scenario B : } & \Delta m_{32}^{2}=\Delta m_{\odot}^{2} \ll \Delta m_{\mathrm{A}}^{2}=\Delta m_{21}^{2} \simeq \Delta m_{31}^{2}
\end{array}
$$

Three mass schemes are capable of providing scenario A, qualitatively shown in Fig. 1:

$$
\begin{array}{ccc}
\text { Scheme A I: } & m_{3} \gg m_{2} \gg m_{1}: & m_{3} \simeq \sqrt{\Delta m_{31}^{2}}, m_{2} \simeq \sqrt{\Delta m_{21}^{2}} \\
\text { Scheme A II: } & m_{3} \gg m_{2} \simeq m_{1}: & m_{3} \simeq \sqrt{\Delta m_{31}^{2}}, m_{2} \simeq m_{1} \simeq 0 \\
\text { Scheme A III: } & m_{3} \simeq m_{2} \simeq m_{1} \equiv m_{0}: & 3 m_{0} \simeq 5 \mathrm{eV},
\end{array}
$$

where $m_{0}$ comes from cosmological considerations [15].

For scenario B, however, there are only two possibilities as presented in Fig. 2:

$$
\begin{array}{ccc}
\text { Scheme B I: } & m_{3} \simeq m_{2} \gg m_{1}: & m_{3} \simeq m_{2} \simeq \sqrt{\Delta m_{31}^{2}}, m_{1} \simeq 0 \\
\text { Scheme B II: } & m_{3} \simeq m_{2} \simeq m_{1} \equiv m_{0}: & 3 m_{0} \simeq 5 \mathrm{eV} .
\end{array}
$$

We stress that there are no other possibilities when Eqs. (2) and (6) are used. Scenario A I can be obtained from the see-saw mechanism [16]; A II and B I, i.e. two very close masses and one separated by the others can be a result of mechanisms generating neutrino masses radiatively [17]. Regardless of the concrete scheme, relations (2) and (4) allow to 
Scheme B I

$m_{3} \simeq m_{2}$
Scheme B II

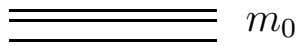

$m_{1}$

Figure 2: The two mass schemes that can accommodate the relation $\Delta m_{32}^{2} \ll \Delta m_{21}^{2} \simeq$ $\Delta m_{31}^{2}$.

get the absolute values of the elements of the MNS matrix [18: In scenario A one finds for a short baseline reactor experiment such as CHOOZ:

$$
P_{e e}^{\mathrm{CHOOZ}}=1-4\left|U_{e 3}\right|^{2}\left(1-\left|U_{e 3}\right|^{2}\right) \sin ^{2} \Delta_{31} / 2
$$

Due to the negative results CHOOZ presents [19], one comes to the conclusion that $\left|U_{e 3}\right|^{2}$ is either very small or close to 1 . Taking into account that the probability $P_{e e}^{\odot}$ for solar neutrinos is significantly lower than 1 , leads to a small value of $\left|U_{e 3}\right|^{2}$ [9]. In that case one has

$$
P_{e e}^{\odot}=1-4\left|U_{e 1}\right|^{2}\left|U_{e 2}\right|^{2} \sin ^{2} \Delta_{21} / 2 .
$$

For oscillations of atmospheric $\nu_{\mu}$ 's into $\nu_{\tau}$ 's one finds

$$
P_{\mu \tau}^{\mathrm{A}}=4\left|U_{\mu 3}\right|^{2}\left|U_{\tau 3}\right|^{2} \sin ^{2} \Delta_{31} / 2 .
$$

In obtaining this equation we have assumed that the oscillation triggered by $\Delta m_{21}^{2}$ washes out. This is the case for $L(\mathrm{~km}) / E(\mathrm{GeV}) \ll 10^{5}$ so that it is advisable to use the throughgoing muons data set of SuperKamiokande (SK). Equations (11) and (12) together with the unitarity of the MNS matrix are now used to get the absolute values of all elements [18]. As the mass and mixing parameters we use the best fit points from [20] for solar neutrinos and for the SK through-going muon sample the values from [21]. The numbers 
used are the following:

$$
\left(\Delta m_{21}^{2}\left(\mathrm{eV}^{2}\right), 4\left|U_{e 1}\right|^{2}\left|U_{e 2}\right|^{2}\right)=\left\{\begin{array}{cc}
\left(5.4 \cdot 10^{-6}, 6.0 \cdot 10^{-3}\right) & \text { SAMSW } \\
\left(1.8 \cdot 10^{-5}, 0.76\right) & \text { LAMSW } \\
\left(8.0 \cdot 10^{-11}, 0.75\right) & \text { VO } \\
\left(\Delta m_{31}^{2}\left(\mathrm{eV}^{2}\right), 4\left|U_{\mu 3}\right|^{2}\left|U_{\tau 3}\right|^{2}\right)= & \left(1.0 \cdot 10^{-2}, 0.78\right)
\end{array}\right.
$$

Note the unusual high value for the atmospheric mass scale. We give now the resulting mixing and mass matrices, starting with scenario A and commenting on scenario B later.

\subsection{The case $\left|U_{e 3}\right| \neq 0$}

The value $\Delta m_{31}^{2}=1.0 \cdot 10^{-2} \mathrm{eV}^{2}$ corresponds in CHOOZ's exclusion plot of [19] to $\left|U_{e 3}\right|^{2} \lesssim$ 0.04. Translating this in Eq. (幽 leads to $\left|s_{3}\right| \lesssim 0.19$ and $\left|c_{3}\right| \gtrsim 0.98 \simeq 1$. This leads to the following mixing matrices:

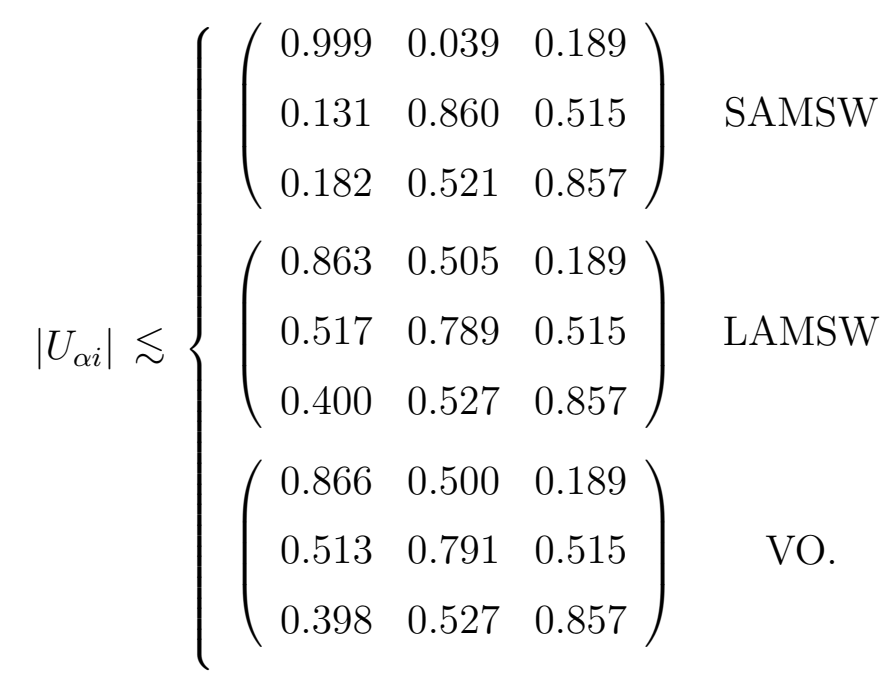

Here we have used that $\left|U_{e 1}\right| \geq\left|U_{e 2}\right|$ as it is necessary for the MSW solutions and inspired by the CKM matrix - $\left|U_{\tau 3}\right| \geq\left|U_{\mu 3}\right|$. The possibility $\left|U_{e 2}\right| \geq\left|U_{e 1}\right|$ is equivalent to an exchange of the first and second column, as $\left|U_{\mu 3}\right| \geq\left|U_{\tau 3}\right|$ is to an exchange of the second and third row.

\subsection{The case $\left|U_{e 3}\right| \simeq 0$}

The observed smallness of $\left\langle m_{e e}\right\rangle$ as measured in $0 \nu \beta \beta$ [22 and the absence of $\nu_{e}$ mixing in atmospheric oscillations has lead many authors (see e. g. [9, 10, 18, 23]) to the assumption $\left|U_{e 3}\right| \simeq 0$. In addition, it can also be related (together with bi-maximal mixing) with the observed flatness in $L / E$ of $\mathrm{SK}^{\prime} \mathrm{s} e$-like events [24]. Since also the phases $\lambda_{1,2}$ in Eq. (4) do 
not contribute to oscillations, this means that there is no observable $\mathrm{CP}$ violation in any oscillation experiment. The mixing matrix now reads:

$$
\left|U_{\alpha i}\right| \lesssim\left\{\begin{array}{ccc}
\left(\begin{array}{ccc}
0.999 & 0.039 & 0 \\
0.033 & 0.856 & 0.515 \\
0.020 & 0.515 & 0.857
\end{array}\right) & \text { SAMSW } \\
\left(\begin{array}{ccc}
0.863 & 0.505 & 0 \\
0.433 & 0.740 & 0.515 \\
0.260 & 0.445 & 0.857
\end{array}\right) & \text { LAMSW } \\
\left(\begin{array}{ccc}
0.866 & 0.500 & 0 \\
0.429 & 0.742 & 0.515 \\
0.258 & 0.446 & 0.857
\end{array}\right) & \text { VO. }
\end{array}\right.
$$

The matrices are of course very similar to the ones derived in 18] where the method was first presented.

\subsection{Scenario B}

Scenario B can be easily obtained from scenario A via cyclic permutation of the columns of the mixing matrices. Hence, the cases to distinguish are $\left|U_{e 1}\right|^{2} \neq 0,\left|U_{e 1}\right|^{2} \simeq 0,\left|U_{e 2}\right|^{2} \geq$ $(\leq)\left|U_{e 3}\right|^{2}$ (the " $\leq$ " case only for the vacuum solution) and $\left|U_{\mu 1}\right|^{2} \geq(\leq)\left|U_{\tau 1}\right|^{2}$. With the two scenarios $\mathrm{A}$ and $\mathrm{B}$ and the possibilities for ordering the mixing matrix elements we have a total of 80 different mass matrices, 48 for scenario A and 32 for scenario B. From these 80 matrices, 48 are stemming from nondegenerate mass schemes. We will show that the 80 reduces to 57 .

\section{Results for $\left\langle m_{\alpha \beta}\right\rangle$}

As shown, the different possibilities are equivalent to exchanges of rows or columns of the mixing matrices. The same holds for the resulting mass matrices. For example, the difference of the $\left|U_{\tau 3}\right| \geq\left|U_{\mu 3}\right|$ case and the $\left|U_{\tau 3}\right| \leq\left|U_{\mu 3}\right|$ case translates into an exchange of $\left\langle m_{\alpha \tau}\right\rangle$ with $\left\langle m_{\alpha \mu}\right\rangle$ with $\alpha=e, \mu$. Replacing $\left|U_{e 1}\right| \geq\left|U_{e 2}\right|$ with $\left|U_{e 1}\right| \leq\left|U_{e 2}\right|$ has no effect on $\left\langle m_{\alpha \beta}\right\rangle$ as long as $\left|U_{e 3}\right| \neq 0$ and generally in the degenerate schemes and in $\mathrm{A}$ II and B I. This, with the appropriate permutations mentioned above, holds for scenario $\mathrm{B}$ as well. Evidently, the degenerate schemes give the same result for $\left\langle m_{\alpha \beta}\right\rangle$ in both scenarios. Therefore from all 80 possible mass matrices only 57 survive, 14 degenerate and 43 nondegenerate ones. We give now for the nondegenerate cases and for all three solar 
solutions the bounds of our results:

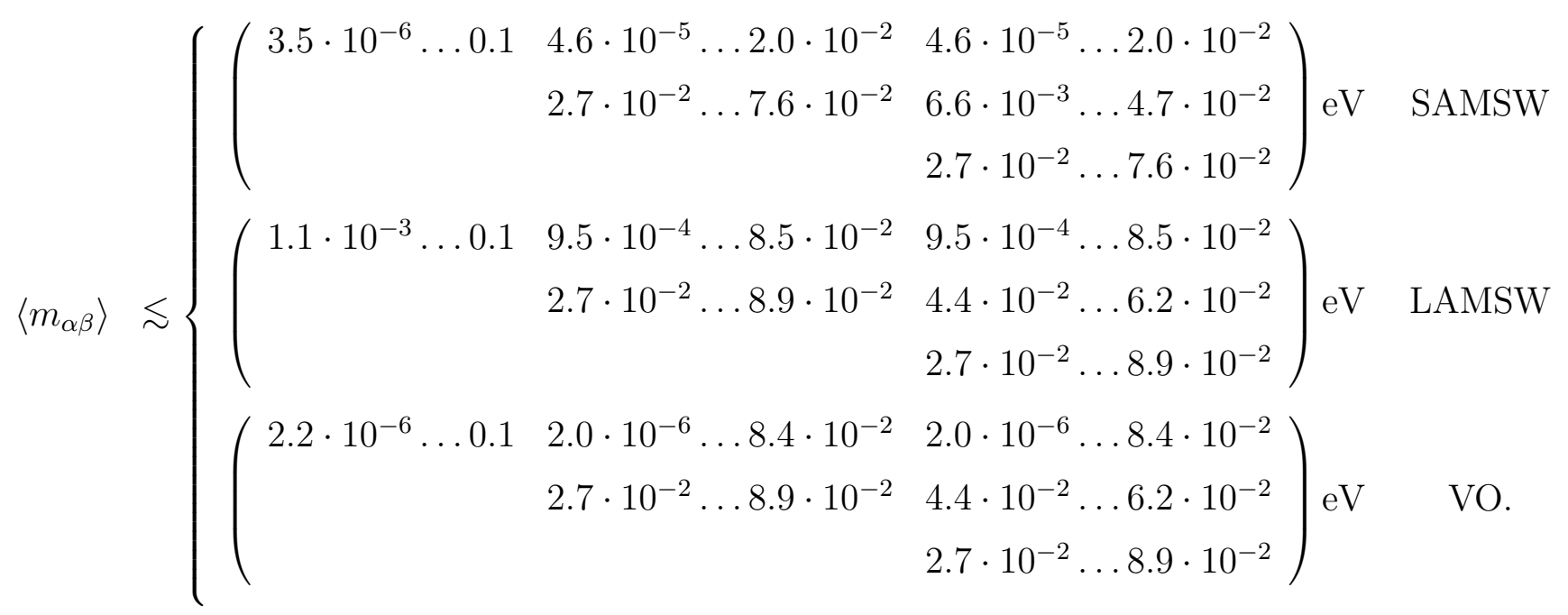

In the scheme (A II, $\left|U_{e 3}\right| \simeq 0$ ) there are zeros as solutions for $\left\langle m_{e \alpha}\right\rangle$, which means values much smaller than the atmospheric scheme, i.e. possibly in the range of $10^{-3} \ldots 10^{-4} \mathrm{eV}$, e.g.

$$
\left\langle m_{e e}\right\rangle \leq\left|U_{e 1}\right|^{2} m_{1}+\left|U_{e 2}\right|^{2} m_{2}+\left|U_{e 3}\right|^{2} m_{3}=m_{1} \ll \sqrt{\Delta m_{\mathrm{A}}^{2}} .
$$

The same can happen in scheme A I, where always a contribution of a value much smaller than the solar scheme can be present. These cases reflect the fact that the smallest mass eigenvalue is never known. For the degenerate scheme the solutions for VO and LAMSW are almost identical:

$$
\left\langle m_{\alpha \beta}\right\rangle \lesssim\left\{\begin{array}{l}
\left(\begin{array}{rrr}
1.67 \ldots 1.73 & 0.74 \ldots 1.57 & 0.74 \ldots 1.57 \\
& 1.67 \ldots 1.93 & 1.47 \ldots 1.77 \\
& & 1.67 \ldots 1.95
\end{array}\right) \text { eV LAMSW and VO } \\
\left(\begin{array}{lll}
1.67 \ldots 1.73 & 0.07 \ldots 0.61 & 0.07 \ldots 0.61 \\
& 1.67 \ldots 1.73 & 1.47 \ldots 1.52 \\
& & 1.67 \ldots 1.73
\end{array}\right) \mathrm{eV} \text { SAMSW. }
\end{array}\right.
$$

The values $>m_{0}$ are explained by the violation of unitarity of the mixing matrices.

\subsection{Properties of the mass matrices}

We start with scenario A: In general, for the hierarchical schemes, $\left\langle m_{\mu \mu}\right\rangle$ is the biggest, $\left\langle m_{e e}\right\rangle$ the smallest entry in $\left\langle m_{\alpha \beta}\right\rangle$. The difference can be up to 4 orders of magnitude (VO and SAMSW, scheme A I, $\left|U_{e 3}\right|^{2} \simeq 0$ ). Entries in the electron row of $\left\langle m_{\alpha \beta}\right\rangle$ are smaller than the other elements. The degenerate scheme has always $\left\langle m_{e e}\right\rangle \simeq\left\langle m_{\mu \mu}\right\rangle \gtrsim\left\langle m_{e \mu}\right\rangle$. In

addition, the $\left|U_{e 3}\right|^{2} \neq 0$ case delivers higher values for all elements of $\left\langle m_{\alpha \beta}\right\rangle$. Scheme III 
gives higher numbers than scheme I which in turn gives higher values than scheme II.

In scenario B all entries are usually in the same order of magnitude yet somewhat higher than in scenario A. The element $\left\langle m_{e e}\right\rangle$, which is the most natural candidate for experimental access, is the only one which is significantly higher, at least one order of magnitude. It

is always bounded by $\sqrt{\Delta m_{\mathrm{A}}^{2}}$, see the next section. As said before, the degenerate scheme gives the same numbers in both scenarios. Two typical matrices show most of the mentioned points, the first for scheme (A I, LAMSW, $\left|U_{e 1}\right| \geq\left|U_{e 2}\right|,\left|U_{\mu 3}\right| \geq\left|U_{\tau 3}\right|$ and $\left|U_{e 3}\right|=0$ ) while the second is for (B II, VO, $\left|U_{e 2}\right| \geq\left|U_{e 3}\right|,\left|U_{\mu 1}\right| \geq\left|U_{\tau 1}\right|$ and $\left|U_{e 3}\right| \neq 0$ ):

$$
\begin{gathered}
\left\langle m_{\alpha \beta}\right\rangle \lesssim\left(\begin{array}{rrr}
3.5 \cdot 10^{-6} & 4.6 \cdot 10^{-5} & 7.7 \cdot 10^{-5} \\
& 7.4 \cdot 10^{-2} & 4.5 \cdot 10^{-2} \\
& & 2.8 \cdot 10^{-2}
\end{array}\right) \mathrm{eV} \\
\quad\left\langle m_{\alpha \beta}\right\rangle \lesssim\left(\begin{array}{rrr}
1.73 & 1.28 & 1.56 \\
& 1.95 & 1.77 \\
& & 1.92
\end{array}\right) \mathrm{eV} .
\end{gathered}
$$

\subsection{Electron neutrino mass}

From inspection of Eq. (16) one sees that the only element accessible to present or near future experiments, $\left\langle m_{e e}\right\rangle$, has the broadest spectrum, a lucky coincidence. On the other hand, due to $\left|U_{e 3}\right|^{2} \ll 1,\left\langle m_{e e}\right\rangle$ is always the smallest entry for scenario A. From all 57 matrices 33 different possibilities for $\left\langle m_{e e}\right\rangle$ exist (it does not matter if $\left|U_{\mu 3}\right| \geq\left|U_{\tau 3}\right|$ or vice versa). From these 33 values $\left\langle m_{e e}\right\rangle$ takes only 10 different values, which are worth taking a closer look at since they spread 6 orders of magnitude. Leaving the ones obtained from degenerated schemes aside, since they lie already above the current experimental limit, we have 8 different values out of 25 matrices, spanning 5 orders of magnitude. It is now tempting to assume that these 8 values have potential to distinguish between the different solar solutions. This is unfortunately not the case:

As a result of relation (2) many schemes have the same value for $\left\langle m_{e e}\right\rangle$, for example (A I, SAMSW, $\left|U_{e 1}\right| \geq\left|U_{e 2}\right|$ and $\left|U_{e 3}\right| \neq 0$ ), (A II, all solar solutions, $\left|U_{e 1}\right| \geq\left|U_{e 2}\right|$ and $\left|U_{e 3}\right| \neq 0$ ) or (A I, VO, $\left|U_{e 2}\right| \geq\left|U_{e 1}\right|$ and $\left|U_{e 3}\right| \neq 0$ ), all yielding $3.6 \cdot 10^{-3} \mathrm{eV}$.

In addition, for scenario $\mathrm{B}$ a peculiarity occurs, namely the bound always takes the same value, regardless of the solar solution:

$$
\left\langle m_{e e}\right\rangle_{\mathrm{B}} \leq\left|U_{e 1}\right|^{2} m_{1}+\left|U_{e 2}\right|^{2} m_{2}+\left|U_{e 3}\right|^{2} m_{3} \simeq m_{3} \simeq \sqrt{\Delta m_{\mathrm{A}}^{2}} .
$$

In the nondegenerate schemes of scenario $\mathrm{B}$, this value of $\left\langle m_{e e}\right\rangle$ is always the largest entry in $\left\langle m_{\alpha \beta}\right\rangle$. Hence a positive signal in a neutrinoless double beta decay experiment will not be able to distinguish between different solar solutions, if nature has chosen scenario B for its neutrinos and an analysis of this kind is used. In addition, when scenario A is realized it will be extremely challenging to distinguish the precise form of the mass and mixing 
scheme as well as to tell which solar solution is the right one. What also complicates the analysis is that, as mentioned above, the contribution of the neglected $m_{1}$ to $\left\langle m_{e e}\right\rangle$ can very well be in the order of $10^{-3} \mathrm{eV}$, making a definite statement somewhat difficult.

Nevertheless, scenario B is only a factor 2 from current experimental limits away, lying well within access in updates of the ${ }^{76} \mathrm{Ge}$ experiment. Although we used a somewhat high value for $\Delta m_{\mathrm{A}}^{2}$ this specific situation seems to be the only realistic candidate for experimental detection. In the next section we will discuss the possibilities of detecting a process sensitive on $\left\langle m_{\alpha \beta}\right\rangle$ in more detail.

\section{Lepton-number and -flavor violating processes and Majorana neutrinos}

For this section it is important to stress again the difference between direct bounds, i.e. considering processes that depend on the respective matrix element and indirect bounds obtained in the present paper, i.e. using oscillation data and unitarity of the MNS matrix. As not surprising, neutrinoless double beta decay is the best examined process triggered by Majorana neutrinos, resulting in a limit of $\left\langle m_{e e}\right\rangle \leq 0.2 \mathrm{eV}$ 22]. The electron-muon element $\left\langle m_{e \mu}\right\rangle$ can be inferred from muon-positron conversion in sulfur nuclei. Theoretical estimations from [25] together with the PDG [26] limit of the branching ratio give a limit of $\left\langle m_{e \mu}\right\rangle \leq 0.4(1.9) \mathrm{GeV}$, when the final state proton pairs are in spin singlet (triplet) state, respectively. The very same diagram as for $0 \nu \beta \beta$ can be applied to other processes like $K^{+} \rightarrow \pi^{-} \mu^{+} \mu^{+}$, which has an experimental branching ratio limit of $1.5 \cdot 10^{-4}$ [27]. Taking the calculation of [28], one finds a limit of $\left\langle m_{\mu \mu}\right\rangle \leq 1.1 \cdot 10^{5} \mathrm{GeV}$. Another process depending on $\left\langle m_{\mu \mu}\right\rangle$ is $\mu^{-} \mu^{+}$conversion in Titanium, discussed in [29]. Instead of nuclear captions or rare decays it was shown in [30], that in principle one can use high-energy scattering processes - in this case tri-muon production at fixed target neutrino-nucleon experiments - to get a bound on $\left\langle m_{\mu \mu}\right\rangle$. Without worrying too much about experimental cuts a limit of $\left\langle m_{\mu \mu}\right\rangle \lesssim 10^{4} \mathrm{GeV}$ was obtained. In 31] this procedure was applied to existing HERA data and generalized to the process

$$
e^{+} p \rightarrow \overline{\nu_{e}} \alpha^{+} \beta^{+} X \text { with }(\alpha \beta)=(e \tau),(\mu \tau),(\mu \mu) \text { and }(\tau \tau),
$$

giving for the first time direct limits on the tau-sector of the mass matrix. Another direct way to obtain information about the tau sector of $\left\langle m_{\alpha \beta}\right\rangle$ might be $B^{+} \rightarrow X^{-} \tau^{+} \alpha^{+}$with $\alpha=e, \mu$ or $\tau$ and $X=\pi, K, D, \ldots$. In total, the current situation for bounds deduced from processes directly depending on $\left\langle m_{\alpha \beta}\right\rangle$ reads:

$$
\left\langle m_{\alpha \beta}\right\rangle \lesssim\left(\begin{array}{ccc}
2 \cdot 10^{-10} & 0.4(1.9) & 4.2 \cdot 10^{3} \\
& 4.0 \cdot 10^{3} & 4.4 \cdot 10^{3} \\
& & 2.0 \cdot 10^{4}
\end{array}\right) \mathrm{GeV}
$$

A spread over 14 orders of magnitude can be seen. An improvement of the values is surely advisable. The somewhat unusual way to use high-energy scattering as done in [30, 31] is 
highly compatible with decay analyses: For example, assuming that a branching ratio for $K^{+} \rightarrow \pi^{-} \mu^{+} \mu^{+}$of about $9.2 \cdot 10^{-8}$ (BR for $K^{+} \rightarrow \pi^{+} \mu^{+} \mu^{-}$, [32]) can be achieved would result in a limit of $\left\langle m_{\mu \mu}\right\rangle \lesssim 3.5 \cdot 10^{3} \mathrm{GeV}$, almost the same number as from HERA data, which itself will be improved by luminosity and energy updates.

There are other Majorana induced $\Delta L \neq 0$ processes, which are at present however not experimentally accessible: Running an $e^{+} e^{-}$collider in $e^{-} e^{-}$mode could give rise to the "inverse neutrinoless double beta decay" $e^{-} e^{-} \rightarrow W^{-} W^{-}$33. The same could be done for a $\mu \mu$ collider or even a possible $e \mu$ machine. For the case we are interested in $\left(s \gg m_{i}^{2}\right)$ the cross section reads 34

$$
\sigma\left(\alpha^{-} \beta^{-} \rightarrow W^{-} W^{-}\right) \simeq \frac{G_{F}^{2}}{4 \pi}\left\langle m_{\alpha \beta}\right\rangle^{2} \simeq 4.30 \cdot 10^{-17}\left(\frac{\left\langle m_{\alpha \beta}\right\rangle}{\mathrm{eV}}\right)^{2} \mathrm{fb},
$$

leaving no prospects for detection, since the cross section is in the order of $10^{-20}\left(10^{-16}\right)$ $\mathrm{fb}$ for hierarchical (degenerate) scheme.

For the sake of completeness one has to add a few words on cancellation of terms in $\left\langle m_{\alpha \beta}\right\rangle$. From Eqs. (3) and (6) it is clear that our bounds are insensitive on the phases of the mixing elements. See [13] for more details on what one could learn from the different phase dependence of $\left\langle m_{e e}\right\rangle,\left\langle m_{e \mu}\right\rangle$ and $\left\langle m_{\mu \mu}\right\rangle$. The Majorana nature brings additional complications via the intrinsic $\mathrm{CP}$-parities $\eta_{i}^{\mathrm{CP}}$ of the mass eigenvalues. Even for $\mathrm{CP}$ invariance one can write 35]

$$
\left\langle m_{\alpha \alpha}\right\rangle=\left.\left|\sum\right| U_{\alpha i}\right|^{2} m_{i} \eta_{i}^{\mathrm{CP}} \mid
$$

making destructive interference in the respective amplitudes possible. Then one can assume mass matrices in flavor space which prohibite special entries in $\left\langle m_{\alpha \beta}\right\rangle$, e.g.

$$
\left\langle m_{\alpha \beta}\right\rangle=\left(\begin{array}{cc}
0 & m \\
m & 0
\end{array}\right), m \geq 0 .
$$

with eigenvalues $\pm m$, first introduced to conserve $L_{e}-L_{\mu}$. Here, the requirement $m_{i} \geq 0$ can be saved by making the mixing matrix elements complex, leading again to cancellation. However, as long as no evidence for a nonvanishing element of $\left\langle m_{\alpha \beta}\right\rangle$ is found, we have no chance to decide which of the above possibilities is realized by nature. Yet, if in direct mass searches for, say, the $\nu_{e}$ a result of a few $\mathrm{eV}$ is found, or the degenerate scheme is somehow verified, one could give bounds on the phases and thus restrict different models.

\section{Conclusions}

Tables 1 (hierarchical scheme) and 2 (degenerate) summarize our results for the different elements of the mass matrices, together with their maximal values obtained from our estimations, the process sensitive to the respective element, a ratio with respect to the relevant standard model process (see below) and a number which indicates how far away 


\begin{tabular}{c|c|c|c|c|c} 
Element & $\max .(\mathrm{eV})$ & Process & Ratio & $\frac{\text { Theory }}{\text { Data }}$ & $\frac{\text { direct }}{\text { indirect }}$ \\
\hline \hline$\left\langle m_{e e}\right\rangle$ & 0.1 & $0 \nu \beta \beta$ & $T_{1 / 2}^{0 \nu} \geq 2.3 \cdot 10^{26} \mathrm{y}$ & 0.25 & 2.0 \\
\hline$\left\langle m_{e \mu}\right\rangle$ & $8.5 \cdot 10^{-2}$ & ${ }^{32} \mathrm{~S}\left(\mu^{-}, e^{+}\right)^{32} \mathrm{Si}$ & $\begin{array}{c}4.5 \cdot 10^{-29} \\
1.8 \cdot 10^{-30}\end{array}$ & $\begin{array}{c}5.0 \cdot 10^{-20} \\
2.0 \cdot 10^{-21}\end{array}$ & $\begin{array}{c}4.7 \cdot 10^{9} \\
2.2 \cdot 10^{10}\end{array}$ \\
\hline$\left\langle m_{e \tau}\right\rangle$ & $8.5 \cdot 10^{-2}$ & $e^{+} p \rightarrow \overline{\nu_{e}} e^{+} \tau^{+} X$ & $3.2 \cdot 10^{-31}$ & $4.0 \cdot 10^{-28}$ & $4.9 \cdot 10^{13}$ \\
\hline$\left\langle m_{\mu \mu}\right\rangle$ & $2.6 \cdot 10^{-2}$ & $K^{+} \rightarrow \pi^{-} \mu^{+} \mu^{+}$ & $6.5 \cdot 10^{-35}$ & $4.4 \cdot 10^{-31}$ & \multirow{2}{*}{$1.5 \cdot 10^{14}$} \\
\hline$\left\langle m_{\mu \tau}\right\rangle$ & $6.2 \cdot 10^{-2}$ & $e^{+} p \rightarrow \overline{\nu_{e}} \mu^{+} \mu^{+} X$ & $3.8 \cdot 10^{-31}$ & $4.9 \cdot 10^{-28}$ & \\
\hline$\left\langle m_{\tau \tau}\right\rangle$ & $8.9 \cdot 10^{-2}$ & $e^{+} p \rightarrow \overline{\nu_{e}} \tau^{+} \tau^{+} X$ & $1.6 \cdot 10^{-31}$ & $2.0 \cdot 10^{-28}$ & $7.1 \cdot 10^{13}$ \\
\hline
\end{tabular}

Table 1: Element of the mass matrix in hierarchical schemes together with its maximal value, a process sensitive to the mass and a ratio with respect to the appropriate standard model process. Note that these highest values come only from scenario B I. Scenarios A I and II have typically mass values one or two orders of magnitude lower, thus the numbers in the last three columns are 2 to 4 orders worse.

we are from detecting the process and thus having access to the element. Also given is the ratio of our indirect bound with the previous direct limits from Eq. (22).

As the ratio we use for the $K$ decay the branching ratio and for the HERA processes (21) the quotient of the respective cross section with the usual standard model charged current process of $\sigma\left(e^{+} p \rightarrow \overline{\nu_{e}} X, Q^{2}>200 \mathrm{GeV}^{2}\right) \simeq 30.3 \mathrm{pb}\left[36\right.$. For $\left\langle m_{e e}\right\rangle$ we give the half-life obtained with the matrix elements from 37] for ${ }^{76} \mathrm{Ge}$.

Theory/Data is a measure for how close (better: how far away) we are from detection of the respective process. We use for $\left\langle m_{e e}\right\rangle$ the current experimental limit for $T_{1 / 2}^{0 \nu}\left({ }^{76} \mathrm{Ge}\right)$ divided by our bound, for the $K$ decay our result divided by the current measured BR limit and for the HERA processes the cross section times the mean value of the luminosity analyzed by H1 38] and ZEUS [39] in searches for isolated lepton events, $\mathcal{L}_{e^{+}}=42.1 \mathrm{pb}^{-1}$. Note the almost hierarchical structure from ee to $\tau \tau$ processes. Theory/Data is a number which characterizes how difficult it is to investigate the respective effective mass. A value less than $10^{-3}$ to $10^{-4}$ for a given $\left\langle m_{\alpha \beta}\right\rangle$ cannot be regarded as accessible in laboratory experiments, even with very positive upgrade assumptions. The numbers show that no element other than $\left\langle m_{e e}\right\rangle$ provides a realistic chance of accession. Regarding scenario A, the highest value obtained for $\left\langle m_{e e}\right\rangle$ is $4.7 \cdot 10^{-3} \mathrm{eV}$, about the limit achievable in the most positive assumption of the GENIUS sensitivity of $2 \cdot 10^{-3} \mathrm{eV}$ at $68 \% \mathrm{C}$. L. for a 10 year run with 10 tons of enriched germanium [40]. Note however that we used the best-fit points of typical oscillation experiment analyses, which of course do not need to be the final answer. However, our value $\Delta m_{\mathrm{A}}^{2}=0.01 \mathrm{eV}^{2}$, the best-fit point for through-going muons at SK, is just the maximum of typical general analyses (see 21] for details) and therefore the values can be regarded as a realistic indication. In scenario $\mathrm{B}\left\langle m_{e e}\right\rangle$ is bounded by the atmospheric mass scale, thus lying well within the range of next generation neutrinoless double beta decay experiments. A detection of a $\left\langle m_{e e}\right\rangle$ in the range of the atmospheric 


\begin{tabular}{c|c|c|c|c|c} 
Element & max. $(\mathrm{eV})$ & Process & Ratio & $\frac{\text { Theory }}{\text { Data }}$ & $\frac{\text { direct }}{\text { indirect }}$ \\
\hline \hline$\left\langle m_{e e}\right\rangle$ & 1.73 & $0 \nu \beta \beta$ & $T_{1 / 2}^{0 \nu} \geq 7.6 \cdot 10^{23} \mathrm{y}$ & 75.7 & 8.7 \\
\hline$\left\langle m_{e \mu}\right\rangle$ & 1.57 & ${ }^{32} \mathrm{~S}\left(\mu^{-}, e^{+}\right)^{32} \mathrm{Si}$ & $\begin{array}{c}1.5 \cdot 10^{-26} \\
6.1 \cdot 10^{-28}\end{array}$ & $\begin{array}{c}1.7 \cdot 10^{-17} \\
6.7 \cdot 10^{-19}\end{array}$ & $\begin{array}{c}2.5 \cdot 10^{8} \\
1.2 \cdot 10^{9}\end{array}$ \\
\hline$\left\langle m_{e \tau}\right\rangle$ & 1.57 & $e^{+} p \rightarrow \overline{\nu_{e}} e^{+} \tau^{+} X$ & $1.1 \cdot 10^{-28}$ & $1.4 \cdot 10^{-25}$ & $2.7 \cdot 10^{12}$ \\
\hline$\left\langle m_{\mu \mu}\right\rangle$ & 1.93 & $K^{+} \rightarrow \pi^{-} \mu^{+} \mu^{+}$ & $3.0 \cdot 10^{-32}$ & $2.0 \cdot 10^{-28}$ & $7.7 \cdot 10^{12}$ \\
\hline$\left\langle m_{\mu \tau}\right\rangle$ & 1.77 & $e^{+} p \rightarrow \overline{\nu_{e}} \mu^{+} \mu^{+} X$ & $1.8 \cdot 10^{-28}$ & $2.2 \cdot 10^{-25}$ & \\
\hline$\left\langle m_{\tau \tau}\right\rangle$ & 1.95 & $e^{+} p \rightarrow \overline{\nu_{e}} \tau^{+} \tau^{+} X$ & $1.3 \cdot 10^{-28}$ & $1.6 \cdot 10^{-25}$ & $2.5 \cdot 10^{12}$ \\
\hline
\end{tabular}

Table 2: Same as previous table for the mass limits obtained from the degenerate mass scheme. Note that the highest values come from the LAMSW or VO solution respectively.

scale would rule out the hierarchical schemes in scenario A.

Direct/indirect shows the ratio of the indirect limits obtained here and the direct limits from Eq. (22). This number can be as high as $10^{14}$. The indirect bounds obtained in this paper are more stringent by this number.

The tables show that the clarification of the question whether neutrinos are Dirac or Majorana particles might have to be postponed. Present and foreseeable experimental possibilities are far beyond verifying some of the given branching ratios or cross sections. This is shown by the ratios in the Theory/Data column. In conclusion, we used a three neutrino framework and studied the range of the elements of the effective mass matrix using all possibilities for the mass and mixing schemes. It turned out that $\left\langle m_{e e}\right\rangle$ has the broadest range of all elements but also is in scenario A the smallest entry and in the nondegenerate schemes of scenario B the highest entry. in general is the smallest value of $\left\langle m_{\alpha \beta}\right\rangle$. We may summarize the situation in saying that if scenario $\mathrm{A}$ is realized little hope should one have, whereas scenario B provides a realistic chance of being probed, leaving neutrinoless double beta decay, the "gold-plated process" of lepton-number/flavor violation. The nice possibility of deciding which solution to the solar neutrino problem is realized is unfortunately not given, but this question will be clarified anyway in ongoing and forthcoming oscillation experiments. For a degenerate scheme the bounds are higher than current experimental limits so that a cancellation of terms in $\left\langle m_{e e}\right\rangle$ as discussed at the end of Section 4 has to occur, provided nature has chosen this scheme. Regarding the other elements of $\left\langle m_{\alpha \beta}\right\rangle$ we showed that there is no possibility to investigate processes depending on them. Nevertheless, the improvement with respect to the previous direct bounds is up to 14 orders of magnitude.

Note added: When this paper was finished, Ref. [41] appeared which gives new limits on two elements of $\left\langle m_{\alpha \beta}\right\rangle$. 


\section{Acknowledgments}

I thank S. M. Bilenky, M. Flanz, E. A. Paschos and K. Zuber for helpful discussions. This work has been supported in part by the "Bundesministerium für Bildung, Wissenschaft, Forschung und Technologie", Bonn under contract number 05HT9PEA5. Financial support from the Graduate College "Erzeugung und Zerfälle von Elementarteilchen" at Dortmund university is gratefully acknowledged.

\section{References}

[1] Y. Fukuda et al. (SuperKamiokande Collaboration), Phys. Rev. Lett. 81, 1562 (1998).

[2] R. Becker-Szendy et al. (IMB Collaboration), Nucl. Phys. B (Proc. Suppl.) 38, 331 (1995),

M. Ambrosio et al. (MACRO Collaboration), Phys. Lett. B 434, 451 (1998),

W. W. M. Allison et al. (Soudan 2 Collaboration), Phys. Lett. B 449, 137 (1999).

[3] K. S. Harata et al. (Kamiokande Collaboration), Phys. Rev. Lett. 77, 1683 (1996),

W. Hampel et al. (GALLEX Collaboration), Phys. Lett. B 388, 384 (1996),

D. N. Abdurashitov et al. (SAGE Collaboration), Phys. Rev. Lett. 77, 4708 (1996),

B. T. Cleveland et al. (Homestake Collaboration), Astrophys. J. 496, 505 (1998),

Y. Fukuda et al. (SuperKamiokande Collaboration), Phys. Rev. Lett. 81, 1158 (1998), erratum ibid. 81, 4279 (1998).

[4] C. Athanassopolous et al. (LSND Collaboration), Phys. Rev. C 58, 2489 (1998).

[5] K. Zuber, Phys. Rep. 305, 295 (1998),

G. G. Raffelt, W. Rodejohann hep-ph/9912397.

[6] L. Wolfenstein, Phys. Rev. D 17, 2369 (1978),

S. P. Mikheyev, A. Yu. Smirnov, Yad. Fiz. 42, 1441 (1985).

[7] B. Zeitnitz et al. (KARMEN Collaboration), Prog. Nucl. Part. Phys. 40, 169 (1998).

[8] Z. Maki, M. Nakagawa, S. Sakata Prog. Theo. Phys. 28, 870 (1962).

[9] S. M. Bilenky et al., Phys. Rev. D 57, 6981 (1998).

[10] V. Barger, K. Whisnant, Phys. Lett. B 456, 194 (1999),

H. Georgi, S. L. Glashow, preprint hep-ph/9808293,

M. Czakon, J. Gluza, M. Zralek, preprint hep-ph/9906381,

F. Vissani, JHEP 9906:22 (1999),

S. M. Bilenky et al. preprint hep-ph/9907234.

[11] H. Nishiura, Phys. Lett. B 157, 442 (1985),

T. Fukuyama, K. Matsuda, H. Nishiura, Mod. Phys. Lett. A 14, 433 (1999). 
[12] S. M. Bilenky, C. Giunti, W. Grimus, Progr. Partl. Nucl. Phys. 43, 1 (1999).

[13] T. Fukuyama, K. Matsuda, H. Nishiura, Phys. Rev. D 57, 5844 (1998);

T. Fukuyama et al., hep-ph/0003055.

[14] S. M. Bilenky, J. Hosek, S. T. Petcov, Phys. Lett. B 94, 495 (1980).

[15] J. R. Primack, A. Klypin, Nucl. Phys. B (Proc. Suppl.) 51, 30 (1996).

[16] M. Gell-Mann, P. Ramond, R. Slansky in Supergravity, p. 315, edited by F. Nieuwehuizen and D. Friedman, North Holland, Amsterdam, 1979,

T. Yanagida, Proc. of the Workshop on Unified Theories and the Baryon Number of the Universe, KEK, Japan 1979,

R. N. Mohapatra, G. Senjanovic, Phys. Rev. Lett. 44, 912 (1980).

[17] A. Zee, Phys. Lett. B 93, 389 (1980),

K. S. Babu, Phys. Lett. B 203, 132 (1988).

[18] C. Giunti, hep-ph/9802201.

[19] M. Apollonio et al. (CHOOZ Collaboration), Phys. Lett. B 420, 397 (1998).

[20] J. N. Bahcall, P. I. Krastev, A. Yu. Smirnov, Phys. Rev. D 58, 096016 (1998).

[21] M. C. Gonzalez-Garcia, hep-ph/9910494, Talk given at the Xth Baksan School "Particles and Cosmology", 19-25 April 1999, Baksan Valley, Kabardino-Balkaria, Russia.

[22] L. Baudis et al., Phys. Rev. Lett. 83, 41 (1999).

[23] V. Barger, S. Pakvasa, T. J. Weiler, K. Whisnant, Phys. Lett. B 437, 107 (1998),

A. J. Baltz, A. S. Goldhaber, M. Goldhaber, Phys. Rev. Lett. 81, 5730 (1998),

H. Fritzsch, Z. Xing, Phys. Lett. B 440, 313 (1998),

R. N. Mohapatra, S. Nussinov, Phys. Rev. D 60, 013002 (1999),

G. Altarelli, F. Feruglio, Phys. Lett. B 439, 112 (1998).

[24] D. V. Ahluwalia, Mod. Phys. Lett. A 13, 2249 (1998),

I. Stancu, D. V. Ahluwalia, Phys. Lett. B 460, 431 (1999).

[25] M. Doi, T. Kotani, E. Takasugi, Prog. Theor. Phys. Suppl. 83, 1 (1985).

[26] Review of Particle Properties, C. Caso et al., Eur. Phys. J. C 3, 1 (1998).

[27] L. S. Littenberg, R. E. Shrock, Phys. Rev. Lett. 68, 443 (1992).

[28] J. Abad, J. G. Esteve, A. F. Pacheco, Phys. Rev. D 30, 1488 (1984).

[29] J. H. Missimer, R. N. Mohapatra, N. C. Mukhopadhyay, Phys. Rev. D 50, 2067 (1994).

[30] M. Flanz, W. Rodejohann, K. Zuber, hep-ph/9907203, to appear in Eur. Phys. J C. 
[31] M. Flanz, W. Rodejohann, K. Zuber, Phys. Lett. B 473, 324 (2000), erratum ibid. 480, 418 (2000).

[32] H. Ma et al., hep-ex/9910047.

[33] D. London, hep-ph/9907419, Talk given at 2nd International Conference Physics Beyond the Standard Model: Beyond the Desert 99: Accelerator, Nonaccelerator and Space Approaches, Ringberg Castle, Tegernsee, Germany, 6-12 June 1999.

[34] G. Belanger et al., Phys. Rev. D 53, 6292 (1996).

[35] S. M. Bilenky, S. T. Petcov, Rev. Mod. Phys. 59, 671 (1987).

[36] M. Derrick et al. (ZEUS Collaboration), Z. Phys. C 72, 47 (1996).

[37] A. Staudt, K. Muto, H. V. Klapdor-Kleingrothaus, Europhys. Lett. 13, 31 (1990).

[38] C. Adloff et al. (H1 Collaboration), Eur. Phys. J. C 5, 575 (1998).

[39] G. Abbiendi et al. (ZEUS Collaboration), hep-ex/9907023.

[40] H. V. Klapdor-Kleingrothaus, hep-ex/9907040.

[41] K. Zuber, Phys. Lett. B 479, 33 (2000). 